\title{
View Constraints: an Interpretation of Integrity Constraints for Security
}

\author{
Patrizia Asirelli \\ Istituto di Elaborazione della Informazione - CNR \\ Via S. Maria, 46, I-56126 Pisa, Italy, tel. +39-50-593477, fax. +39- \\ 50-554342, email: asirelli@iei.pi.cnr.it
}

\begin{abstract}
The problem of Integrity Constraints checking in the area of Logic Databases is considered and some research results are presented in which the notion of View Constraints as restriction on a database emerges. This notion is presented and compared with the general notion of Integrity Constraints. Some examples are given and implementation issues are discussed.
\end{abstract}

\section{Keywords}

Integrity Constraints, Views, Logic/Deductive Databases, Database Integration, Security

\section{INTRODUCTION}

The problem of Integrity Constraints checking in the area of Logic Databases has been considered where Integrity Constraints are interpreted as obligations. Alternatively the same formulae can be interpreted as defining subsets of a given database, View Constraints i.e. Restrictions .

As is apparent in the literature, the particular way it is decided to consider the role of constraints, is often a pragmatic issue, depending on the application at hand. It is generally accepted that constraints express properties of a given database but the differences between the various methods described in the literature generally concern questions of how the constraints are proved to be satisfied by the database (proof methods) and when such proofs should be performed. The differences are often a matter of the meaning assigned to such formula (constraints) with respect to an application domain.

One interesting interpretation of constraints in databases is the ability to define a particular view of the database as a refinement of its relations. The

*work partially funded by Progetti Coordinati CNR-Comitato 12 : 'KINE-Knowledge Integration Environment' and 'Programmazione Logica (Logic Programming)' 
notion of view that we are thinking of allows us to impose a set of restrictions on a database dynamically in order to filter elements that do not satisfy certain properties out of the relations of the database. This is why we can call them restriction constraints.

We claim that this interpretation of constraints, is suitable to consider the problem of security in a deductive database (integration) context.

In fact, the Internet and the World Wide Web capability are showing the need for organizations to access and integrate different sources of information. Future applications are likely to be built by putting together systems developed and managed at different sites. Integration, federation, cooperation etc of information sources or software systems, in general, seems to be a must. Security and privacy are thus becoming more crucial (Jajodia 1996a), (Jajodia 1996b), both for relational and advanced database systems.

Integration of databases is a hot area of research. Interesting proposals for mediating database systems can be found in the literature, both for relational (Silberschatz, Stonebraker and Ullman 1991), (Ullman 1994) and logic/deductive databases (Baral, Kraus and Minker 1991), (Subrahmanian 1994), (Lu et al 1995), (Aquilino et al. 1995), (Asirelli, Renso and Turini 1996), (Aquilino et al. 1997).

Security, within the framework of deductive databases and their integration has also been studied (Bonatti, Kraus and Subrahmanian 1995), (Candan, Jajodia and Subrahmanian 1996).

Here we present a possible use of an integrity constraint checking approach for security. In particular, we consider a method described in (Asirelli, De Santis and Martelli 1985), and its different implementations and extensions (Asirelli, Inverardi and Plagenza 1996), (Aquilino et al. 1995), (Asirelli, Renso and Turini 1996), (Aquilino et al. 1997).

This kind of approach does not deal with the problem of 'correct setting' of Constraints, neither it is concerned with the particular meaning attached to constraints, e.g. 'access constraints'. It is a general framework where the designer defines its own constraints by means of logical formulas that are handled according to a given logical semantics. It is the designer responsibility to define constraints in such a way that they play a specific role e.g. 'access constraints'.

In the following Section 2 we will summarise the background and the results in the literature that have led to our considerations on these two kind of database constraints. Few examples of applications of this concept of constraints as 'restrictions' will be illustrated in Section 3. Compilative and interpretative implementation issues are discussed in section4. Section 5 states our conclusions. 


\section{INTEGRITY CONSTRAINTS AND VIEW CONSTRAINTS}

We deal with constraints on the basis of the following notions:

- Deductive databases considered in this paper refer to the notion of database as the set union of logic clauses in the EDB and in the IDB, i.e. $D B=$ $E D B \cup I D B$ where there is no necessary distinction between base predicates and derived predicates, as is the case for Datalog. Thus, we consider a deductive database as a simple logic program;

- Queries are simply goals that are evaluated in the given DB (LP);

- Constraints are formulae of this kind: $A \rightarrow B_{1}, \ldots, B_{n}$ (Asirelli, De Santis and Martelli 1985),(Kowalski 1995), with the intuitive meaning that: for every ground instance of $A$, say $A \sigma$, there must exist a ground instance of $\left(B_{1} \wedge \ldots \wedge B_{n}\right) \sigma$ which holds.

Various kinds of integrity constraint formulae and verification methods in the area of deductive databases have been proposed by different authors (Abiteboul, Hull and Vianu 1995)(Gallaire, Minker and Nicolas 1982). Some authors denote integrity constraint formulae as denials $\leftarrow A_{1}, \ldots, A_{n}$ (Lloyd and Topor 1985), (?), with the following intuitive semantics: for every ground instance of $A_{1} \wedge \ldots \wedge A_{n}$, say $\left(A_{1} \wedge \ldots \wedge A_{n}\right) \sigma$, there must exist at least one $A_{i} \sigma$ which does not hold.

As summarised by Kowalski (1995), all integrity constraints checking methods proposed so far, have tried to give a different formal characterisation to the informal semantics of integrity constraints. These include a:

- theoremhood view (Lloyd and Topor 1985), (Asirelli, De Santis and Martelli 1985), where the constraints formulas have to be logically entailed by the database, i.e. $D B \mid=I C$;

- consistency view (?), where the theory obtained by the union of the database and the constraints must have a model, i.e. $D B \cup I C$ has a model and furthermore, an

- epistemic (Reiter 1990) or metalevel view (?), where, as far as we understand, constraints are always satisfied by the database, i.e. the database system is such that the database will never reach a state where the constraints are violated.

Furthermore, the different integrity checking methods have followed different strategies. In fact, they can either:

i) assume that the database is consistent, check at update time that the update preserves consistency and accept or reject it. This is the case for applications where the database has to be 'protected', e.g. banking or business databases in general; 
ii) allow for updates, even those generating an inconsistent database, periodically perform a consistency checking and, in case of inconsistency, try to restore the consistency in various ways; some authors also propose changing the constraints accordingly (Di Nitto and Tanca 1996), this is considered acceptable e.g. for 'scientific' databases where the rules change as new discoveries are obtained;

or

iii) pre-compile the integrity constraints and the database into a new database $D B^{\prime}$ such that, by definition, $D B^{\prime}$ will satisfy the integrity constraints. In this case a query is answered in $D B^{\prime}$ and thus the situation is similar to i).

We concentrate on this last approach which, in our opinion, is well suited for applications where a common database has to be viewed by different users, but some knowledge is hidden to certain users e.g. for security reasons.

This approach falls into that theoremhood view of constraints, in which the constraints are logically entailed not by the original database but by the database that results from applying a restriction (modification) imposed by the constraints. i.e.

$$
D B / m I C==>D B^{\prime} \mid=I C
$$

However, in our opinion this approach also falls into the epistemic view, and thus leads to considering the constraints not just as Integrity Constraints (obligations) as in the common use of the term, but as defining subsets of a given database (View Constraints). Within this interpretation, the theory describing the integrity constraint formulae can be considered as a view on the database, i.e. as a specification of the relevant facts. This means that IC are here dealt with in a more general way. They can be different for different users, they can be used for security(Bertino, Jajodia and Samarati 1995), and in particular for secrecy maintenance or for availability (Jajodia 1996a) and (Jajodia 1996b). That is, for hiding information or else for showing only that information that satisfies certain criteria and, furthermore, to define some exceptions to database rules thus implementing Default Reasoning, as in (Kowalski and Sadri 1990).

We believe that this interpretation of constraints as Views (restrictions), is suitable for considering the problem of security in a deductive database context and, even more, in a deductive database integration context.

The situation can be summarised by the following three examples:

In this first example, no constraint is specified and thus a query to the database will produce the only answer that can be derived from the database. 


\section{Example 1}

$E D B: \quad \mathrm{P}(\mathrm{a}, \mathrm{b}) \quad \mathrm{P}(\mathrm{b}, \mathrm{c}) \quad \mathrm{P}(\mathrm{c}, \mathrm{d})$ $Q(a, n) \quad Q(s, m)$

$I D B: \quad \mathrm{R}(\mathrm{X}) \leftarrow \mathrm{Q}(\mathrm{X}, \mathrm{Y}), \mathrm{P}(\mathrm{X}, \mathrm{Z})$

$I C:$ none

query : $R(X)$ only $\mathbf{R}(\mathbf{a})$ is computed

Here we show a database that satisfies its Integrity Constraints, i.e. all answers that are 'computable' in the database are correct wrt constraints.

\section{Example 2}

$E D B: \begin{array}{lll}\mathrm{P}(\mathrm{a}, \mathrm{b}) & \mathrm{P}(\mathrm{b}, \mathrm{c}) & \mathrm{P}(\mathrm{c}, \mathrm{d}) \\ \mathrm{Q}(\mathrm{a}, \mathrm{n}) & \mathrm{Q}(\mathrm{s}, \mathrm{m}) & \\ \mathrm{R}(\mathrm{a}) & & \end{array}$

$I D B:$ none

$I C$ :

$$
R(X) \rightarrow Q(X, Y), P(X, Z)
$$

integritychecking: OK

The following example shows a situation where Constraints are used as Views (restrictions): only those answers that satisfy the constraints are computed.

\section{Example 3}

$$
E D B: \begin{array}{lll}
\mathrm{P}(\mathrm{a}, \mathrm{b}) & \mathrm{P}(\mathrm{b}, \mathrm{c}) & \mathrm{P}(\mathrm{c}, \mathrm{d}) \\
\mathrm{Q}(\mathrm{a}, \mathrm{n}) & \mathrm{Q}(\mathrm{s}, \mathrm{m}) & \\
\mathrm{R}(\mathrm{a}) & &
\end{array}
$$

$I D B$ :

$$
R(X) \leftarrow Q(X, Y)
$$

$I C: \mathrm{R}(\mathrm{x}) \rightarrow \mathrm{P}(\mathrm{x}, \mathrm{z})$

query: ?R(X) only $\mathbf{R}(\mathbf{a})$ is computed

Thus a theory of 'View Constraint' on a database defines a restriction on the database model. Accordingly, different theories of 'View Constraint' define 
different 'restrictions' of the same model. We can depict this situation as follows:
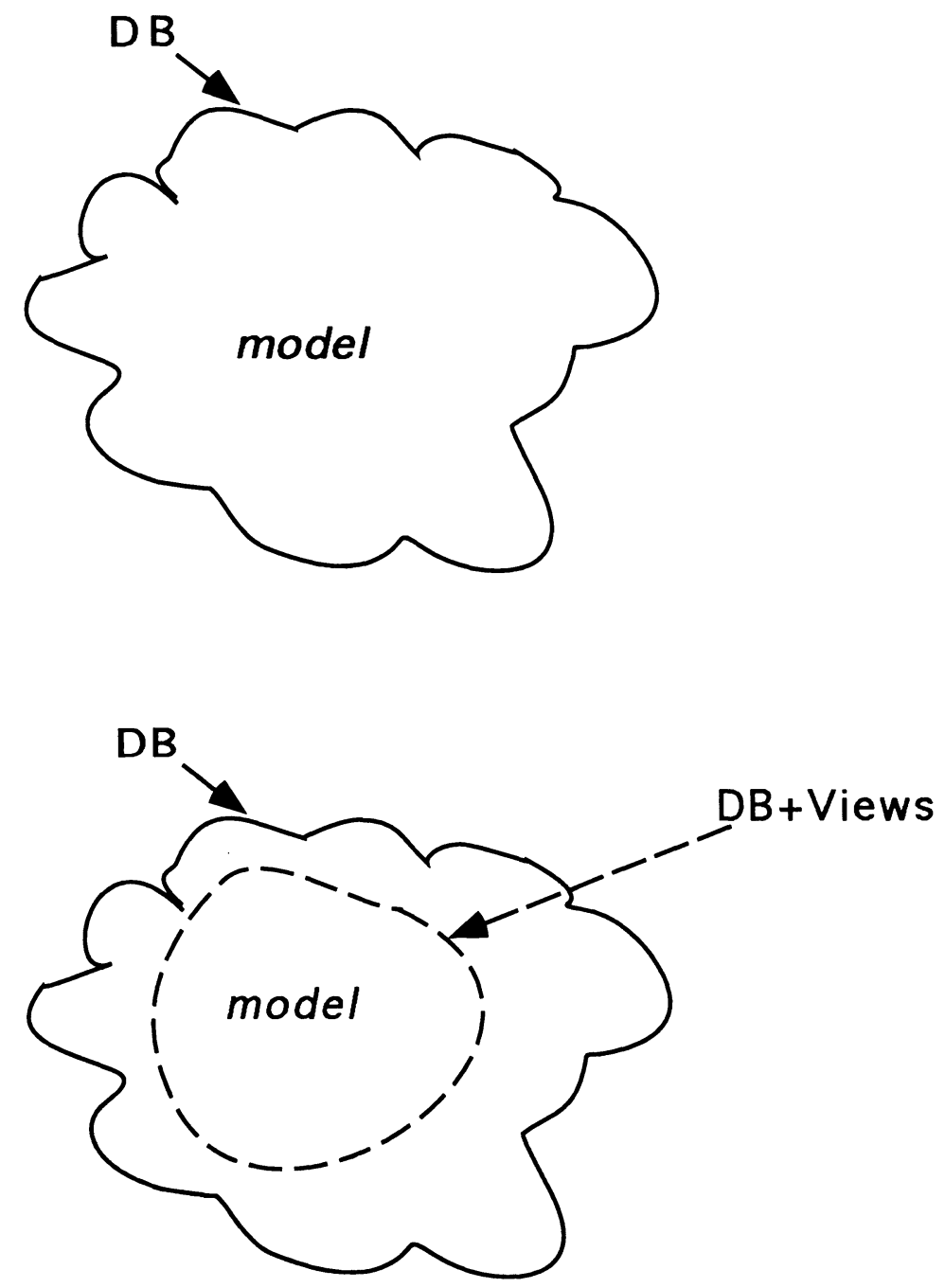


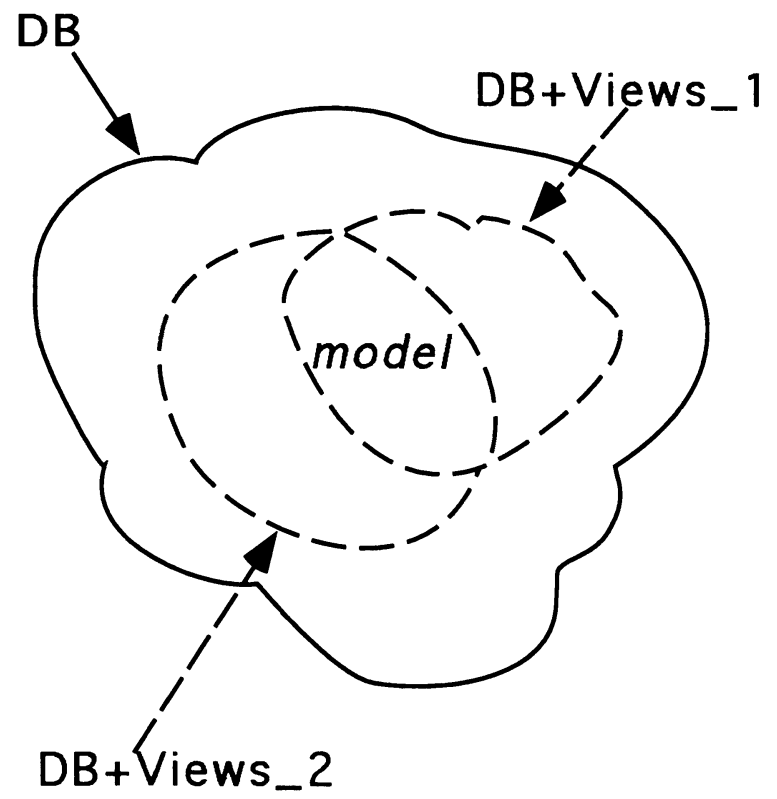

In the first case we have the database and its model; in the second we depict the restriction of this model by one 'View Constraint', while in the third we represent the different restrictions on the same database model imposed by different views.

\section{EXAMPLES}

Few examples of application of the two concepts of Integrity Constraints and View Constraints are going to be sketched.

\subsection{A database of friends and lovers}

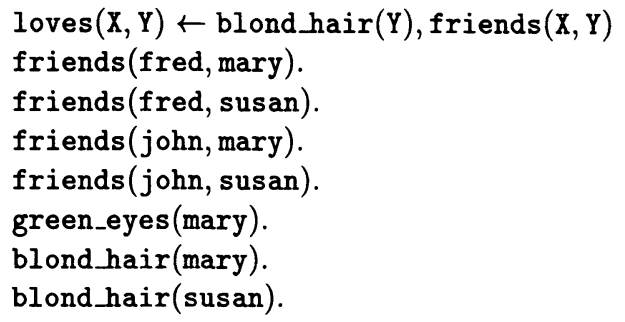

In the above database both john and fred loves at the same time both mary and susan. If there are constraints: 
1) The $D B$ and IC are separated theories

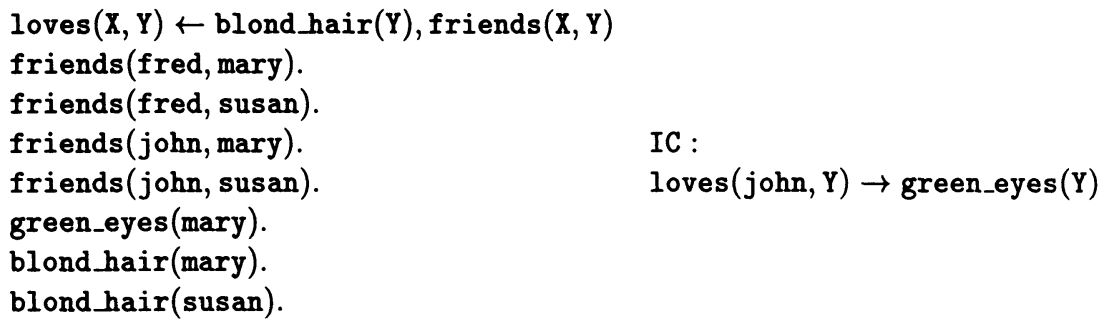

Then, while fred still loves susan and mary, john can only love mary. The set of answers to the database that are correct with respect to constraints is equivalent to the set of answers that can be computed by the following program. See also section 3.3.

2) The $D B$ and IC are merged together

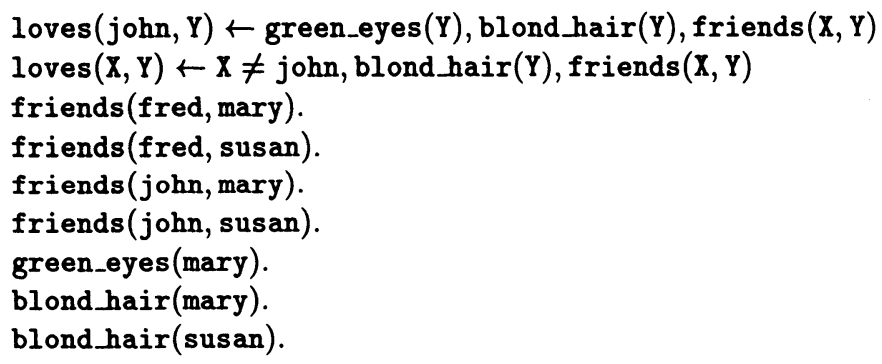

\subsection{A bank database example}

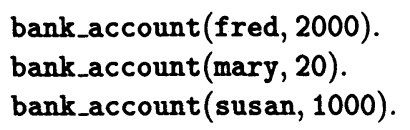

Then everybody can make a query and get to know information about the other accounts. If there are constraints, then:

1) The $D B$ and $I C$ are separated theories

DB :

bank_account(fred, 2000).

bank_account (mary, 20).

IC :

bank_account (susan, 1000).

bank_account $(X, Y) \rightarrow$ authorized $(X, Z)$, director $(Z)$

director(smith). 
Then, no one can get to know anything, since no one is authorized. The same results could be obtained by the following program:

\section{2) The $D B$ and IC are merged together}

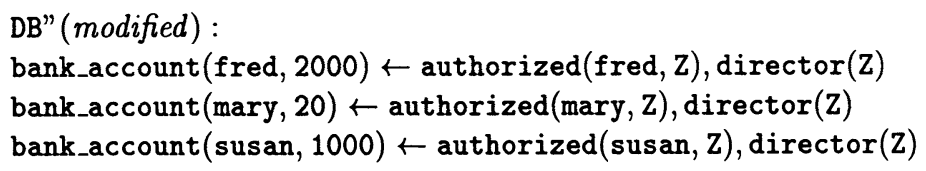

Else, we can formally think to give to each user (fred, susan etc.) a database, personilezed as the following one defined for mary.

\section{3) $A D B$ for Mary}

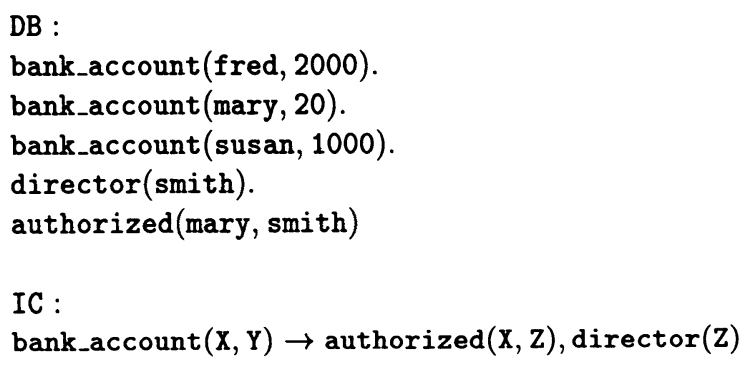

Efficiency can be obtained, of course, by maintaining only a database and adding one constraint, each time, for each user, as it is shown by the alternative approach as in section 3.3. More efficiency can also be obtained at implementation level (see section 4).

\subsection{A Constraint Operator}

The application we are going to present shows how View Constraints can be used for the definition of a 'constraint' operator among logic programs (Aquilino et al. 1995), (Asirelli, Renso and Turini 1996), (Aquilino et al. 1997).

The following example shows the union $(U)$, intersection $(\cap)$ and constraint (/) operators among programs. These program operators have been used to define an approach to the 'semantic integration' of databases (Asirelli, Renso and Turini 1996), where they are used to define 'mediators' (Silberschatz, Stonebraker and Ullman 1991)and (Ullman 1994) programs.

Roughly, the union among two programs is just the collection of all clauses in the two programs. The intersection is given by the set of clauses obtained by unifying the heads of the clauses and putting together the corresponding instances of the bodies. The formal definitions of $U$ and $\cap$ can be found in (Brogi et al. 1994).

The / operator (Aquilino et al. 1995), (Aquilino et al. 1997) defines a pro- 
gram as the result of 'constraining' the definition of predicates in the first program on the basis of the definitions (of the same predicates) contained in the second one.

Example 4 Programs (Databases) Integration

$$
\begin{aligned}
& \text { Database P: Database Q : } \\
& \begin{array}{l}
r(X, Y) \leftarrow s(X) \\
t(a)
\end{array} \quad r(a, Y) \leftarrow t(a) \\
& \mathrm{t}(\mathrm{a}) \quad \mathrm{s}(\mathrm{a}) \\
& \text { Database } P \cup Q: \quad \text { Database } P \cap Q \text { : } \\
& r(X, Y) \leftarrow s(X) \\
& r(a, Y) \leftarrow t(a) \quad r(a, Y) \leftarrow t(a), s(a) \\
& \mathbf{s}(\mathrm{a}) \\
& \text { Database } P / Q \text { : } \\
& r(\mathrm{a}, \mathrm{Y}) \leftarrow \mathrm{s}(\mathrm{a}), \mathrm{t}(\mathrm{a}) \\
& r(X, Y) \leftarrow X \neq Y, s(X) \\
& t(a)
\end{aligned}
$$

Thus, taking into account the in section 3.2 , we are in a better position to implement a 'security' interface to a database. We can handle a single database:

\section{Unic_DB}

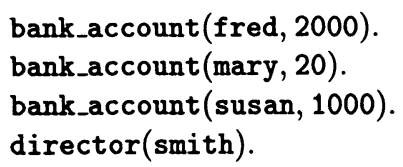

and then have a Constraint Theory:

IC

bank_account $(X, Y) \leftarrow \operatorname{authorized}(X, Z), \operatorname{director}(Z)$

and then to each user give a Database defined as follows:

- mary: (Unic_DB U authorized(mary, smith))/ IC

- fred: (Unic_DB $\cup$ authorized(fred, smith))/ IC

- susan: (Unic_DB $\cup$ authorized(susan, smith))/IC 


\section{IMPLEMENTATIONS}

Two implementation approaches of the notion of View Constraints are sketched. The first approach refers to the works in (Asirelli, De Santis and Martelli 1985) where the constraints are expressed by formulas as in this paper, and (Aquilino et al. 1995), (Aquilino et al. 1997), where they are defined by another deductive database, as it is the case in section 3.3. In this kind of implementations a new database state is computed on the basis of the old one and the Integrity Constraints.

The second implementation approach takes a more dynamic view: It shows the definition of a new refutation procedure (SRP-IC) which is based on SLD. Every answer to a query which is computed by SRP-IC is successful if and only if it is entailed by the database and satisfies the constraints. This procedure is only briefly presented here, while more details can be found in (Asirelli, Inverardi and Plagenza 1996).

\subsection{The 'compilative' approach}

In the first implementation approach, given a database and a set of 'restrictions', a new deductive database is computed, the relations of which are the original ones properly filtered according to the restrictions. Thus, starting from a database and its set of Integrity Constraints (IC), this implementation method merges them into a new database, that corresponds to the original one modified by the formula in the IC. The behaviour of this approach is also shown in section 3.1 where the DB and IC are merged together.

Actually, although the semantics is exactly the same, the algorithm to perform this modification defined in (Asirelli, De Santis and Martelli 1985) (and actually implemented in our database system GEDBLOG) would compute an intermediate predicate, one for each constraint clause. This predicate would then be inserted in the definition of the original predicate. That is, referring to the example in section 3.1, we would get a database such as the following one:

\section{Example 5}

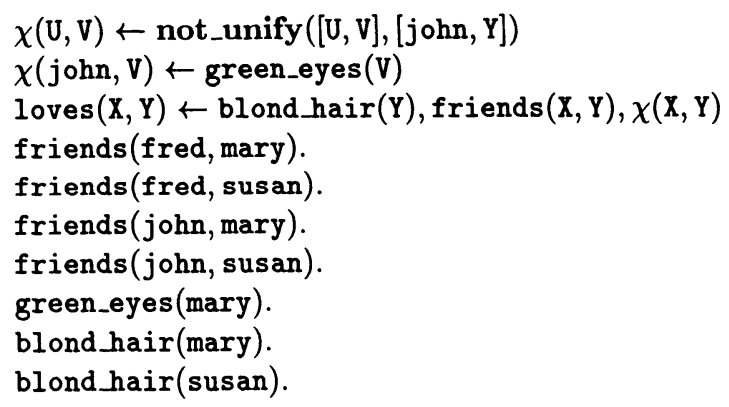


The same use of constraints to restrict the set of possible answers to a query on a database also appears in (Gaasterland et al. 1992). That method also adopts a compilative approach as in the above cited ones and constraints are expressed as denials.

The above methods of implementing view constraints all computes a new DB or Logic Program. While it is 'normal' to recompile a program after an update, when a database is concerned, an update, either to the DB or to the constraints, enforces a (partial) recompilation of the DB. This, in some cases can be dreadful, from the efficiency point of view.

\subsection{The 'interpretative' approach}

The second implementation method would allow the DB to remain the same, while particular properties changes, e.g. from one user to another.

Very roughly the intuition underlying the 'interpretative implementation' method is that:

during the resolution of a query, whenever we have to resolve an instance of an atom $A$, say $A \theta$, and there is a constraint $\bar{A} \rightarrow B_{1}, \ldots, B_{n}$ such that $\bar{A}$ and $A \theta$ unify with m.g.u. $\alpha$, then $\left(B_{1}, \ldots, B_{n}\right) \alpha$ must be added to the obtained subquery.

Thus, as a consequence of applying a constraint, we add the adequately instantiated consequence part of the constraint to the goal obtained by resolving the atom that unifies with the head of the constraint.

Let us consider the following example.

Suppose we have a theory graph which defines two extensional relations, node and edge, and one intentional relation, path, with the straightforward meaning.

\section{Example 6 A Theory for Graphs}

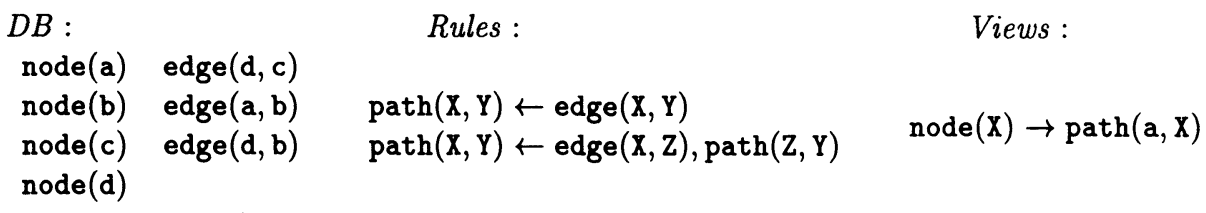

The above view establishes that we accept only nodes that are reachable from node $a$. Now, we show the results of some queries on the theory graph with respect to the constraint.

The query $\leftarrow \operatorname{node}(d)$ violates the constraint, while the query $\leftarrow$ node $(b)$ satisfies it. 


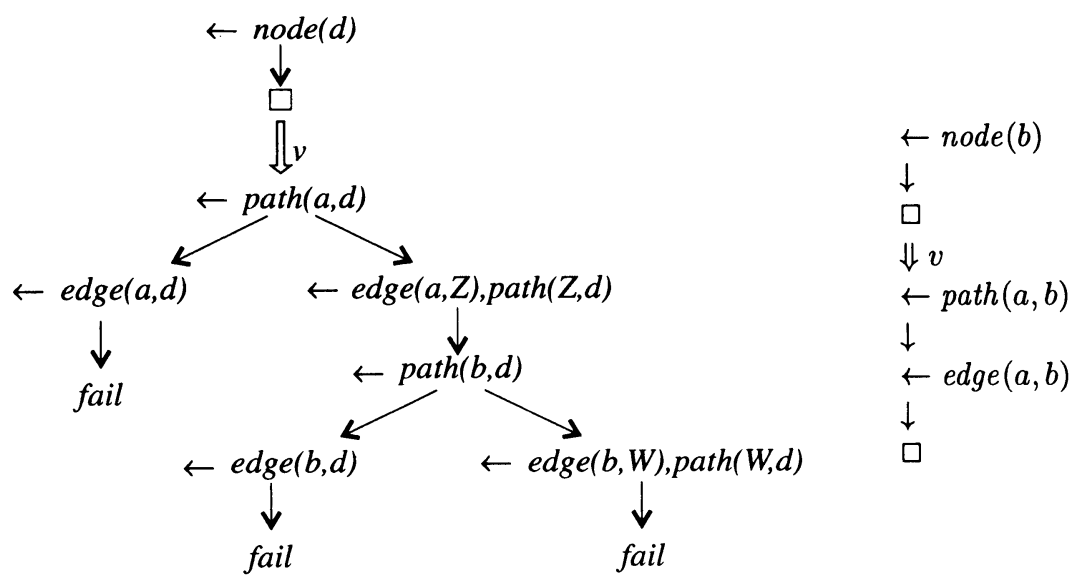

Here, $\downarrow$ represents the usual resolution step by means of rule $r_{i}$ and $\Downarrow$ represents the application of the integrity formula to the goal (selected atom) just resolved (also called the selected-solved atom).

Despite the simplicity of the general idea its application needs a careful treatment and analysis of the various instantiation situations that arise during the resolution due to the unification process, especially if there are many constraints that can be applied at the same time.

This interpretative approach, also is a basis to obtain interoperability and cooperation in the deductive database context. Correspondingly, the compilative approach has been used to define semantic integration of logic databases (Asirelli, Renso and Turini 1996), mediators are defined by means of operators among DB, including the 'constraint' operator). Such an approach to integrate databases has also been used to define integration of Geographical Information Systems (Aquilino, Renso and Turini 1996)

\section{CONCLUSIONS}

We have considered the problem of Integrity Constraints checking in the area of Logic Databases and presented some research results that account for two different notions of constraints. On one hand, constraints are considered as 'obligations' ( Integrity Constraints), on the other they can be considered as defining 'restrictions' on a database ( View Constraints). We claim that the differences among the two notions are often a matter of the meaning assigned to such formulas (constraints) with respect to an application domain. Furthermore we claim that this interpretation of constraints as Views (restrictions), is suitable to consider the problem of security in a deductive database (integration) context. 
We also claim that among various interpretation of constraints in databases the 'View Constraints' one is very interesting since it provides the ability of defining a particular view of the database as a refinement of its relations. This notion allows one, among other possible operations, to impose dynamically a set of restrictions on a database in order to filter elements that do not satisfy certain properties, out of the relations of the database. This means that IC are here dealt with in a more general way. They can be different for different users, they can be used for security and in particular for secrecy maintenance or for availability. That is, for hiding information or else for showing only that information that satisfies certain criteria and, furthermore, to define some exceptions to database rules thus implementing Default Reasoning.

This kind of approach does not deal with the problem of 'correct setting' of Constraints, neither it is concerned with the particular meaning attached to constraints, e.g. 'access constraints'. It is a general framework where the designer defines its own constraints by means of logical formulas that are handled according to the following logical semantics: the final DB is 'consistent' with respect to the constraints, or else, the 'constraints' are 'logical consequences' of the DB. It is the designer responsibility to define constraints in such a way that they play a specific role e.g. 'access constraints'.

Few examples of application of the concept of View Constraints have been illustrated.

Two implementation approaches of the notion of View Constraints are also sketched. A 'compilative' and an 'interpretative' approach. In the compilative implementation of the methods (section 4.1) the 'consistency' of the new (amalgamated) database with respect to the old database is defined by the formal definition of the methods and its semantics (Asirelli, De Santis and Martelli 1985), (Asirelli, Inverardi and Plagenza 1996), (Aquilino et al. 1995), (Aquilino et al. 1997). It is in fact proved that the methods are correct and complete with respect to the stated semantics: no new information is inserted, no 'correct' information is deleted by the methods.

Note that, in this implementation method the 'amalgamated DB' is really computed (new rules and facts are generated, from the old ones and the constraints) while it is not in the 'interpretative approach' (section 4.2).

This work is a first step in the direction of studying this approach more deeply for security purposes. The approach described in (Asirelli, De Santis and Martelli 1985) is implemented in our system GEDBLOG (a deductive DBMS (Asirelli et al. 1994)). We are at present extending it to handle the constraint operator and also we are porting GEDBLOG to work on the Internet so that mediators can be effectively implemented.

We are at present also investigating the use of the interpretative implementation as a basis to obtain interoperability and cooperation in the deductive database context. 


\section{REFERENCES}

Abiteboul, S. Hull, R. and Vianu, V. (1995) Foundations of Databases, Addison-Wesley Pubs.Co.

Aquilino, D. Asirelli, P. Renso, C. and Turini, F. (1995) An Operator for Composing Deductive Databases with Theories of Constraints, in Proc. of Logic programming and Non-Monotonic Reasoning '95, Lecture Notes in Computer Science, 928, Springer-Verlag, Berlin.

Aquilino, D. Asirelli, P. Renso, C. and Turini, F. (1997) Applying Restriction Contraints to Deductive Databases, In Non-determinism in Deductive Databases, (ed. D. Pedreschi and V.S. Subrahmaniam), Annals of Mathematics and Artificial Intelligence, 19(1,2), 3-25.

Aquilino, D. Renso, C. and Turini, F. (1996) Towards Declarative GIS analysis Proc. of the Fourth ACM-GIS Worskhop, Rockville, Maryland USA- November.

Asirelli, P. De Santis, M. and Martelli, M. (1985) Integrity Constraints in Logic Databases, Journal of Logic Programming, 3,221-32.

Asirelli, P. Di Grande, D. Inverardi, P. and Nicodemi, F. (1994) Graphics by a Logic Database Management System, Journal of Visual Languages and Computing, 5, 365-88.

Asirelli, P. Inverardi, P. and Plagenza, G. (1996) Integrity Constraints as Views in Deductive Databases, Proc. of 6th Int. Workshop on Foundations of Models and Languages for Data and Objects, Integrity in Databases, IBFI Schloss Dagstuhl, Germany, September 16-20, Ottovon-Guericke- Universitat MAGDEBURG, Preprint, 4.

Asirelli, P. Renso, C. and Turini, F. (1996) Language Extensions for Semantic Integration of Deductive Databases, Proc. of the International Workshop on Logic In Databases (LID'96), Lecture Notes in Computer Science, 1154, Springer-Verlag, Berlin.

Baral, C. Kraus, S. and Minker, J. (1991) Combining Multiple Knowledge Bases, Transaction on Knowledge and Data Engineering, 3(2), 208220 .

Bertino, E. Jajodia, S. and Samarati, P. (1995) Database security: Research and practice, Information Systems, 20 (7), 537-556.

Bonatti, P. A. Kraus, S. and Subrahmanian, V. S. (1995) Foundations of Secure Deductive Databases, IEEE Transaction on Knowledge and Data Engineering, 7 (3), 406-422.

Brogi, A. Chiarelli, A. Mazzotta, V. Mancarella, P. Pedreschi, D. Renso, C. and Turini, F. (1994) Implementations of Program Composition Operations, Proc. of the Sixth Int'l Symp. on Programming Language Implementation and Logic Programming, (ed. M. Hermenegildo and J. Penjam), Lecture Notes in Computer Science, 844, Springer-Verlag, Berlin.

Candan, K. S. Jajodia, S. and Subrahmanian, V. S. (1996) Secure Mediated 
Databases, International Conference on Data Engineering 1996, 28-37. Di Nitto, E. and Tanca, L. (1996) Dealing with Deviations in DBMSs: An Approach to revise Consistency Constarints, Proc. of 6th Int. Workshop on Foundations of Models and Languages for Data and Objects, Integrity in Databases, IBFI Schloss Dagstuhl, Germany, September 16-20, Otto-von-Guericke- Universitat MAGDEBURG, Preprint, 4.

Gaasterland, T. Godfrey, P. Minker, J. and Novik, L. (1992) A Cooperative Answering System, in Proc. of the Logic Programming and Automated Reasoning Conference, (ed. A. Voronkov), Lecture Notes in Artificial Intelligence, 624, Springer-Verlag, Berlin, 478-480.

Gallaire, H. Minker, J. and Nicolas, J.M. (1982) Advances in Database Theory (vol. II), Proc. of the Workshop on Logical Bases for Databases, , Toulouse.

Jajodia, S. (1996a) Database security and privacy, ACM Computing Surveys, 50th anniversary commemorative issue, 28 (1).

Jajodia, S. (1996b) Managing Security and Privacy of Information, ACM Computing Surveys, 28 (4es), 129-131.

Kowalski, R.A. (1995) Using Meta-logic to Reconcile Reactive with Rational Agents, in Meta-logics and Logic Programming, (ed. K.R. Apt and F. Turini), MIT Press.

Kowalski, R. and Sadri, F. (1990) Logic Programs with Exceptions, Proc. of the 7th Int. Conf on Logic Programming, (ed. DHD Warren and P. Szeredi), Israel, The MIT Press, Cambridge, Mass.

Lloyd, J.W. and Topor, R.W. (1985) A Basis for Deductive Database Systems, in Journal of Logic Programming, 2, 93-109.

$\mathrm{Lu}$, J. J. Moerkotte, G. Schue, J. and Subrahmanian, V. S. (1995) Efficient Maintenance of Materialized Mediated Views, SIGMOD Conference 1995, 340-351.

Reiter, R. (1990) On asking what a database knows, in Computational Logic (ed. J.W. Lloyd) Springer Verlag, Esprit Basic Research Series, 96-113.

987) SAKO87 Sadri, F. and Kowalski, R. (1987) A Theorem-Proving Approach to Database Integrity, in Foundation of Deductive Databases and Logic Programming, (ed. J. Minker), Morgan-Kaufmann, 313-362.

Silberschatz, A. Stonebraker, M. and Ullman, J. D. (1991) Database Systems: Achievements and Opportunities, Communication of the ACM, 34(10), 110-120.

Subrahmanian, V. S. (1994) Amalgamating Knowledge Bases, Transaction On Database System, 19(2), 291-331.

Ullman, J. (1994) High Level Interoperation, Slides, December. 